ANDRÉ Alves CARDOSO ${ }^{1}$

Régis Resende Paulinelu ${ }^{2}$

RUFFO FREITAS-JÚNIOR ${ }^{2}$

Rosemar Macedo Sousa Rahal ${ }^{2}$

TATIANA FERRARI JACINTO

Artigos originais

Palavras-chave

Mama/cirurgia Ginecomastia/cirurgia Ginecomastia/complicações Procedimentos cirúrgicos reconstrutivos/métodos

Homem

Keywords

Breast/ surgery Gynecomastia/surgery Gynecomastia/complications Reconstructive surgical procedures/methods

Man

\title{
Análise comparativa da técnica da incisão em duplo círculo com as técnicas com incisão periareolar e transareolomamilar de correção cirúrgica da ginecomastia
}

\author{
Comparative analysis of the double-circle incision and techniques \\ with periareolar and transareolopapilar incision for the surgical \\ correction of gynecomastia
}

\section{Resumo}

OBJETIVO: comparar a técnica da incisão em duplo círculo (DC) com as técnicas de incisão periareolar (PA) e transareolomamilar (TAM), na correção da ginecomastia. MÉTODOS: foram revisados os prontuários de 34 pacientes com ginecomastia submetidos à correção cirúrgica no Hospital das Clínicas de Goiânia de 1999 a 2004. Os pacientes foram divididos em três grupos, de acordo com a técnica cirúrgica utilizada. Comparamos as variáveis numéricas paramétricas usando o teste de Tukey. Para as variáveis nominais, foi utilizado o teste do $\chi^{2}$, ou o teste exato de Fisher, quando necessário. Considerou-se significante o p<0,05. RESULTADOS: a média de idade dos pacientes foi de 27,9 $(+12,5)$ anos. Foram operadas 43 mamas: 34 unilaterais $(79,1 \%)$ e nove $(20,9 \%)$ bilaterais; $19(44,2 \%)$ pelo DC; $14(32,6 \%)$ com incisão PA; $10(23,3 \%)$ com incisão TAM. A média do tempo de utilização de dreno foi de cinco dias para o DC e um dia para as demais $(p<0,01)$. Foram utilizados drenos de sucção 19 vezes (100\%) para o DC e duas vezes (22\%) na transareolomamilar. Nas outras, utilizaram-se drenos de Penrose $(p<0,01)$. $\bigcirc$ tempo cirúrgico foi significativamente maior para o DC (73 minutos) que para a PA (45 minutos) e a TAM (48 minutos). O DC foi utilizado principalmente em ginecomastias mais volumosas $(p=0,04)$. Quanto às complicações foram observados: três casos de hematomas com a TAM $(33,3 \%)$ e um hematoma $(5,3 \%)$ com o DC ( $<<0,01)$; houve um caso de infecção com a TAM $(11,1 \%)$; duas necroses parciais do mamilo com a DC $(10,5 \%)$; quatro $(21,1 \%)$ cicatrizes alargadas e três $(15,8 \%)$ cicatrizes hipertróficas com o DC (p=0,04); uma inversão do mamilo com ○ TAM (2,4\%). CONCLUSÕES: a técnica do DC é uma boa opção para correção de ginecomastias volumosas, embora exija maior tempo cirúrgico e apresente mais cicatrizes alargadas.

\section{Abstract}

PURPOSE: to compare the double-circle (DC) technique to other techniques, with periareolar (PA) and transverse nippleareolar (TNA) incisions, for the surgical correction of gynecomastia. METHODS: we studied the medical files of 34 patients from the Federal University of Goiás, submitted to the surgical correction of gynecomastia, from 1999 to 2004. Patients were divided according to the surgical technique used. The parametric numeric variables were compared by Tukey test. The $\chi^{2}$ or the Fisher's exact test was used for nominal variables. It was considered significant a p value<0.05. RESULTS: the mean age of the patients was $27.9(+12.5)$ years. There were 43 gynecomastias, 34 unilateral (79.1\%) and nine (20.9\%) bilateral. There were 19 breasts operated (44.2\%) using DC, $14(33.6 \%)$ using PA incision, and $10(23.3 \%)$, TNA incision. The mean drain usage was five days for DC and one day for the others $(p<0.01)$. The suction drain was used in 19 cases (100\%) of DC and two (22\%) in TNA. The other patients used drains of Penrose ( $p<0.01)$. The mean surgical time was significantly larger for DC (73 minutes) than for PA (45 minutes) and for TNA (48 minutes). DC was used mainly in voluminous gynecomastias $(p=0.04)$. The complications consisted in three $(33 \%)$ hematomas in TNA $(p<0.01)$ and one (5\%) in DC; one (1 1\%) infection in TNA; two (10\%) partial necrosis of the nipple in DC; four (21\%) enlarged scars in $D C(p=0.04)$; three (16\%) hypertrofic scars $(p=0.08)$ in $D C$; one $(2 \%)$ inversion of nipple with TNA. CONCLUSIONS: The DC was used often in voluminous gynecomastias. It was a good and secure operation, although it required a more extensive surgical time and had a larger possibility of distended scars.

Correspondência:

Ruffo Freitas-Júnior Primeira Avenida, $s / n-$ Setor Universitárín CEP 74605-050 - Goiânia/GO Fone/Fax: (62) 3945-4769 E-mail: ruffo@medicina.ufg.br

Recebido 12/02/2007

Aceito com modificacōes $23 / 08 / 2007$
Programa de Mastologia do Departamento de Ginecologia e Obstetrícia da Faculdade de Medicina da Universidade Federal de Goiás - UFG - Goiânia (GO), Brasil.

Médico Estagiário do Programa de Mastologia do Departamento de Ginecologia e Obstetrícia da Faculdade de Medicina da Universidade Federal de Goiás - UFG - Goiânia (GO), Brasil.

2 Médico do Programa de Mastologia do Departamento de Ginecologia e Obstetrícia da Faculdade de Medicina da Universidade Federal de Goiás - UFG - Goiânia (GO), Brasil. 


\section{Introdução}

A ginecomastia pode ser definida como o aumento benigno de tecido glandular mamário (ductal e estromal) no indivíduo masculino. Estima-se que cerca de 30 a $50 \%$ da população masculina seja acometida em alguma fase da vida ${ }^{1}$. É mais comum na adolescência e na senectude, e pode ser um processo transitório ${ }^{2,3}$. Pode ser decorrente do uso de medicações ou de outras doenças orgânicas, embora em metade das vezes a causa não seja identificada ${ }^{2,4,5}$. A ginecomastia pode trazer prejuízos estéticos, constrangimento e redução da auto-estima dos pacientes. Deve-se fazer o diagnóstico diferencial com a pseudoginecomastia, por acúmulo de tecido adiposo em indivíduos obesos, e com o carcinoma mamário, em caso de nódulos ${ }^{6}$.

A ginecomastia pode regredir espontaneamente em $25 \%$ das vezes, quando idiopática ou após a correção do problema desencadeante (uso de medicamentos e doenças associadas). A correção cirúrgica é necessária quando não ocorre a regressão ou quando o indivíduo assim o desejar, por questões pessoais ${ }^{3}$.

As técnicas cirúrgicas de correção da ginecomastia mais conhecidas são a técnica periareolar (PA) e a técnica transareolomamilar (TAM $)^{1}$. A técnica do duplo círculo (DC), apesar de pouco difundida, começou a ser utilizada em nosso Serviço há alguns anos, na tentativa de melhorar o campo operatório em casos em que se esperava maior dificuldade técnica, pelo maior volume das mamas, ou quando o paciente deseja corrigir também o tamanho da aréola ${ }^{7,8}$.

O objetivo deste trabalho é comparar a técnica do DC com as demais técnicas mais comuns de correção da ginecomastia, quanto à preferência dos cirurgiões, tempo cirúrgico e ocorrência de complicações.

\section{Métodos}

Nesse estudo, foram avaliados retrospectivamente os prontuários de 34 pacientes com ginecomastia submetidos à correção cirúrgica no Hospital das Clínicas da Universidade Federal de Goiás, entre os anos de 1999 e 2004 . Foram pesquisados dados concernentes à idade dos pacientes, volume mamário, técnica utilizada (DC, PA e TAM), tempo cirúrgico, uso e tipo de dreno, complicações imediatas e tardias, satisfação do paciente e necessidade de reintervenção.

Foram incluídos no estudo todos os homens com ginecomastia há mais de um ano, que não apresentaram regressão espontânea da mesma e que desejavam a correção cirúrgica por motivos estéticos, após explicação dos detalhes e dos riscos principais do procedimento.
Foram investigados na anamnese o uso de medicamentos e a presença de comorbidades. Os exames laboratoriais ficaram a critério do médico assistente, que solicitava exames pré-operatórios rotineiros. Quando havia suspeita de doenças associadas causadoras de ginecomastia, foram investigados cromossomopatias, tumores testiculares, hepatopatia, nefropatia, etc. Como se trata de um estudo retrospectivo, não havia uma rotina estabelecida para a solicitação de exames.

As cirurgias foram realizadas pelo médico especializando em Mastologia, sempre acompanhado por um dos professores, já especialista, com experiência na execução das técnicas. Segue a descrição detalhada das cirurgias:

- descrição da técnica do duplo círculo (Figura 1): os pacientes estavam anestesiados - geralmente sob anestesia peridural e, às vezes, sob anestesia geral. Com o paciente em decúbito dorsal, foram realizadas a anti-sepsia com Povidine ${ }^{\mathrm{R}}$ e a antibioticoprofilaxia com $1 \mathrm{~g}$ de cefalotina ou cefazolina. Os campos foram colocados, marcou-se a pele a ser cortada com o azul de metileno, com dois círculos concêntricos. O primeiro círculo delimitava a região periareolar ou, quando se deseja realizar a redução da aréola, um círculo de 3 a $5 \mathrm{~cm}$ de diâmetro com o mamilo no centro; o segundo círculo, de diâmetro maior que o primeiro, foi calculado de acordo a quantidade de pele que se desejava ressecar, de forma a possibilitar um acesso cirúrgico mais amplo à mama do paciente. Após a remoção do tecido glandular, aproximaram-se os bordos dos dois círculos, eliminando o excesso de pele.

A seguir, foi realizada a decorticação da pele pela manobra de Schwartzman entre os dois círculos. A incisão do parênquima foi realizada em um arco de $120^{\circ}$ sobre a borda inferior do círculo externo, de forma a não ultrapassar $50 \%$ do perímetro do círculo.

A glândula mamária foi ressecada gentilmente, deixando um coxim subcutâneo de 5 a $10 \mathrm{~mm}$ sob o retalho, de forma a não esgarçar ou lesar a derme decorticada, uma vez que o suprimento sangüíneo e a sensibilidade nervosa para o complexo areolomamilar dependem da integridade desta área. Foi realizada a hemostasia rigorosa do leito cirúrgico e instalado dreno de sucção contínua.

A sutura foi iniciada com quatro pontos cardinais unindo as bordas dos dois círculos, invaginando a área decorticada, seguido de sutura com pontos separados tipo Perseu ("U" vertical da aréola e "U" horizontal intradérmico no círculo externo), com o nó voltado internamente ao complexo areolomamilar. Por fim, era 
realizado curativo com micropore, tendo o cuidado de não comprimir o retalho e provocar isquemia.

No pós-operatório, foram utilizados analgésicos e antiinflamatórios. A alta hospitalar normalmente ocorreu no dia seguinte e o dreno foi retirado com cinco dias. Em caso de formação de seroma, o mesmo era puncionado nos retornos ambulatoriais. A sutura normalmente era retirada em 19 dias.

- descrição das técnicas com incisão periareolar e transareolomamilar: o procedimento de anestesia, anti-sepsia, antibioticoprofilaxia e a ressecção glandular foram semelhantes à técnica do DC. A diferença principal foi a incisão na pele, como o próprio nome indica. $\mathrm{Na}$ incisão periareolar, evitou-se ultrapassar com a incisão $50 \%$ da aréola, devido ao risco de necrose. A utilização de dreno ficou a cargo do cirurgião, que decidia, de acordo com a extensão da ressecção glandular e da dificuldade de hemostasia, se colocava um dreno tubular de sucção contínua por cinco dias ou um Penrose número 1 por um dia.

\section{Análise dos dados}

A comparação entre as variáveis numéricas paramétricas relativas aos pacientes operados pelas diferentes técnicas foi feita por meio da média, do desvio padrão e da análise de variância com teste de Tukey. Para a comparação das variáveis nominais, foi utilizado o teste do $\chi^{2}$, ou o teste exato de Fisher, quando necessário. Para a análise estatística, foi considerado como significante $\mathrm{p}<0,05$. O programa utilizado foi o SPSS, versão 11.0.1.

\section{Resultados}

A média de idade dos pacientes operados foi de $27,9( \pm 12,5)$ anos. Não houve diferença significante quanto à média de idade dos pacientes operados com as três técnicas de correção cirúrgica da ginecomastia, sendo de 26,0 $(+13,8)$ anos no DC, 26,0 $(+11,1)$ anos na PA e 35,6 (+12,0) na TAM.

Dos 34 pacientes, nove $(26, \%)$ apresentavam ginecomastia bilateral, totalizando 43 mamas operadas: 19 (44\%) pela técnica do DC; 14 (33\%) pela técnica PA; 10 (23\%) pela TAM.

A freqüência de bilateralidade foi de $12(63,2 \%)$ mamas na DC, quatro $(28,6 \%)$ na PA e duas $(11,1 \%)$ na TAM. Houve uma tendência significante do cirurgião em preferir a técnica do DC em ginecomastias bilaterais, com relação à PA e TAM $\left(\chi^{2}=5,75 ; \mathrm{p}<0,02\right)$.

$\mathrm{O}$ volume de tecido ressecado foi significativamente maior na técnica do DC, mostrando preferência dos ci-
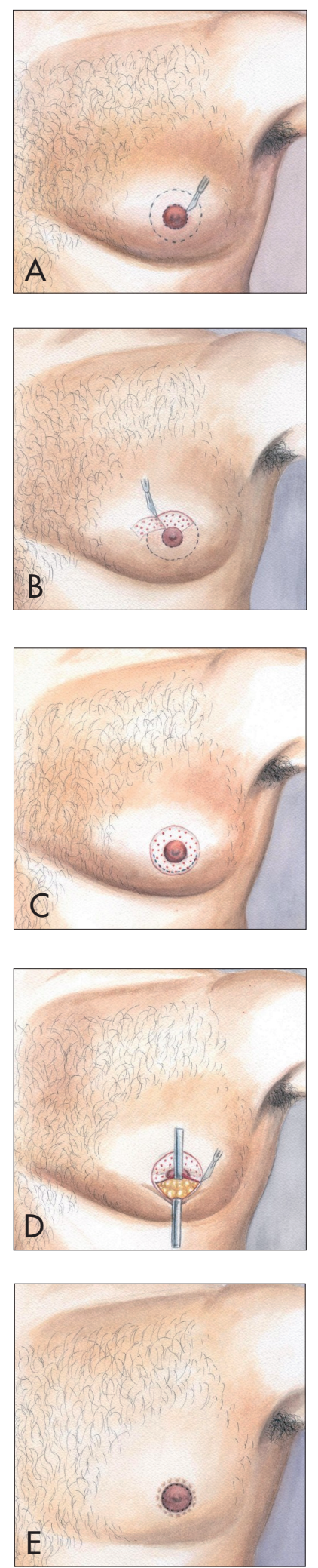

Figura 1 - Técnica do duplo círculo: a. marcação dos dois círculos ao redor do mamilo; b. início da decorticação; c. decorticação completada; d. incisão na borda inferior da área cruenta e ressecção do tecido glandular; e. síntese da pele unindo os dois círculos e resultado final. 
rurgiões em utilizá-la em ginecomastias mais volumosas, como pode ser visto na Tabela 1 . Na análise estatística, o tempo cirúrgico foi significativamente maior para a técnica do DC em comparação à técnica PA e à TAM, como também pode ser visto na Tabela 1 .

O dreno de sucção contínua foi utilizado em 19 (100\%) mamas operadas pela técnica do DC, em nenhuma operada pela incisão PA e em duas $(22,2 \%)$ operadas pela TAM $(\mathrm{p}<0,01)$. Quando não foi utilizado o dreno tubular, foi utilizado o Penrose número 1.

Foram avaliados: presença de hematomas, necessidade de reintervenção por sangramento, infecção, necrose do mamilo, seroma, cicatrizes inestéticas, inversão do mamilo, recidiva da ginecomastia, assimetria entre as mamas ou qualquer outra complicação descrita no prontuário. Ocorreu algum tipo de intercorrência em $24(60,5 \%)$ casos, sendo mais comum o seroma. A análise comparativa das complicações entre as diferentes técnicas pode ser vista na Tabela 2 . Houve três $(33,3 \%)$ casos de pequenos hematomas, com resolução clínica, na técnica TAM, e nenhum caso nas outras técnicas $(\mathrm{p}<0,01)$. Ocorreu apenas um caso de hematoma volumoso, com necessidade de reintervenção, na técnica do DC (NS). Todos os sete $(36,9 \%)$ casos de alargamento ou hipertrofia da cicatriz ocorreram na técnica do DC, o que foi estatisticamente significante. Apesar disso, o único paciente que desejou realizar uma segunda cirurgia por insatisfação com o resultado estético havia sido operado pela incisão TAM.

\section{Discussão}

A ginecomastia pode regredir espontaneamente em muitos casos. Quando a mesma é persistente e traz prejuízo estético ou psicológico ao paciente, é necessária a correção. Existem tratamentos alternativos, como o uso de medicações ou de radioterapia, mas o tratamento cirúrgico é geralmente o mais utilizado ${ }^{7-10}$. Existe uma tendência na literatura de utilizar-se a lipoaspiração no tratamento da ginecomastia. Os resultados são melhores em pacientes com mamas mais adiposas ${ }^{11-13}$. Em nosso Hospital, não empregamos essa técnica.

No período estudado, todos os casos foram resolvidos com a excisão da glândula mamária, seja por meio do acesso PA, TAM ou pela confecção do DC. Na análise estatística, percebeu-se que a técnica do DC foi a preferida pelos cirurgiões de nosso Hospital em ginecomastias mais volumosas, apesar do maior tempo cirúrgico e da possibilidade de cicatrizes mais alargadas. Provavelmente isso é devido ao melhor campo operatório proporcionado nesta técnica. Ela permite ainda a correção do tamanho da aréola, que freqüentemente pode estar aumentada nos casos de ginecomastia. Uma técnica semelhante vem sendo proposta em caso de ginecomastias volumosas em outras séries ${ }^{14,15}$.

A idade dos pacientes com ginecomastia em nosso estudo foi semelhante a outras séries da literatura, com dois grupos principais: pacientes jovens entre 15 e 25 anos e um grupo menor de pacientes de meia idade ${ }^{11}$.

Tabela 1 - Análise comparativa do tempo cirúrgico e das características da peça cirúrgica entre as diferentes técnicas de correção cirúrgica da ginecomastia.

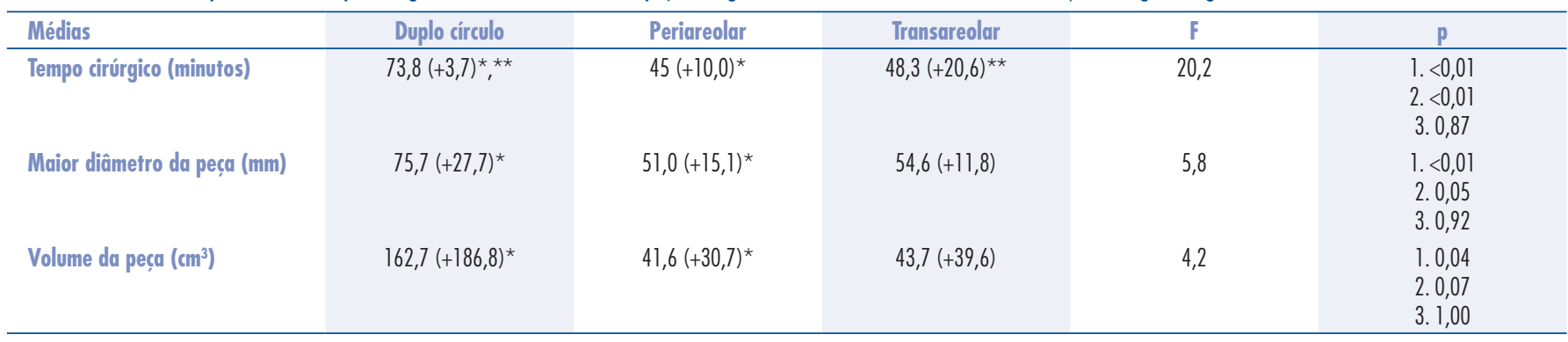

1. Duplo círculo versus periareolar; 2 . duplo círculo versus transareolomamilar; 3 . periareolar versus transareolomamilar; ${ }^{*}$,* diferença significante; dados reportados como média \pm desvio padrão.

Tabela 2 - Comparação da freqüência de complicações com 0 uso das diferentes técnicas de correção cirúrgica da ginecomastia (n e porcentagem).

\begin{tabular}{|c|c|c|c|c|c|}
\hline Freqüências & Duplo círculo & Periareolar & Transareolar & $\chi^{2}$ & $p$ \\
\hline Hematoma com resolução & - & $-\star \star$ & $3(33,3) *$, & 7,93 & $<0,01$ \\
\hline Reintervenção por hematoma & $1(5,3)$ & - & - & 0,95 & 0,33 \\
\hline Infecção & - & - & $1(11,1)$ & 2,5 & 0,11 \\
\hline Necrose parcial do mamilo & $2(10,5)$ & - & - & 1,95 & 0,16 \\
\hline Seroma & $4(21,1)$ & $5(35,7)$ & $2(22,2)$ & 0,08 & 0,78 \\
\hline Cicatriz alargada & $4(21,1)^{\star}, \star \star$ & - & $-\star \star$ & 4,01 & 0,04 \\
\hline
\end{tabular}

Não houve nenhum caso de recidiva da ginecomastia, nem de assimetria marcante; ${ }^{*},{ }^{\star \star}$ diferença significante. 
A idade dos pacientes não interferiu na técnica cirúrgica utilizada.

O uso de drenos de sucção foi significativamente maior para os casos operados pela técnica DC. A instalação e a permanência de qualquer dreno estiveram na dependência da decisão do cirurgião, de acordo com o grau de ressecção glandular e dificuldade de hemostasia. Mesmo com o uso do dreno de sucção, houve um caso de hematoma volumoso, exigindo reintervenção. Acreditamos que o risco de hematoma esteja mais relacionado ao volume da ginecomastia e da ressecção do que propriamente à técnica cirúrgica.

Na técnica da incisão TAM, houve maior freqüência de hematomas pequenos com resolução clínica. Como o estudo foi retrospectivo e não controlado, não sabemos o porquê dessa diferença, mas pode estar relacionada ao cirurgião que utilizou a técnica, ao volume da mama (intermediário entre as corrigidas pelo DC e PA), ao não uso de dreno de sucção, ou a um campo operatório mais restrito. Para descobrir o motivo, seria necessário um estudo prospectivo randomizado comparando as técnicas.

O tempo cirúrgico foi significativamente maior para o DC, pois é necessária uma decorticação da pele entre os dois círculos e maior quantidade de pontos de sutura. Além disso, as ginecomastias escolhidas pelos cirurgiões para usar esse método foram as de maior volume. Provavelmente, a escolha foi devida a melhor exposição do leito cirúrgico e maior facilidade para a realização da hemostasia.

A incidência de complicações foi semelhante à de outras séries na literatura ${ }^{12,13,16,17}$. Houve, porém, maior número de cicatrizes alargadas com o DC. Porém, nenhum dos pacientes quis se submeter à revisão da cicatriz. $\mathrm{O}$ único paciente que decidiu ser reoperado por insatisfação com o resultado estético havia sido operado pela técnica de incisão TAM. Não houve caso de recidiva da ginecomastia, nem de assimetria marcante. Ocorreram dois casos de necrose parcial do mamilo com a técnica do DC e nenhum caso com as demais técnicas. Apesar de a diferença não ser significante, é possível que possa vir a ser com o aumento do número de casos, pois, com o DC, é realizada uma decorticação extensa da pele ao redor do mamilo. É um dos elementos a ser lembrado na decisão da escolha da técnica ideal para cada paciente.

Podemos, então, concluir que a técnica do duplo círculo é uma boa opção para possibilitar a correção de ginecomastias volumosas, embora necessite maior tempo cirúrgico e apresente maior possibilidade de cicatrizes alargadas.

\section{Referências}

1. Gikas P, Mokbel K. Management of gynaecomastia: an update. Int J Clin Pract. 2007;61 (7):1209-15.

2. Ismail AA, Barth JH. Endocrinology of gynaecomastia. Ann Clin Biochem. 2001;38(Pt 6):596-607

3. Wiesman IM, Lehman JA Jr, Parker MG, Tantri MD, Wagner DS, Pedersen JC. Gynecomastia: an outcome analysis. Ann Plast Surg. 2004;53(2):97-101.

4. Strub C. Gynecomastia and HIV infection. Schweiz Rundsch Med Prax. 2003;92(1 1):507-9.

5. Afflitto L. Drug-induced gynecomastia. Plast Surg Nurs. 2000;20(3): 189-90, 194.

6. Voulliaume D, Vasseur C, Delaporte T, Delay E. An unusual risk of liposuction: liposuction of a malignant tumor. About 2 patients. Ann Chir Plast Esthet. 2003;48(3): 187-93.

7. Persichetti P, Berloco M, Casadei RM, Marangi GF, Di Lella F, Nobili AM. Gynecomastia and the complete circumareolar approach in the surgical management of skin redundancy. Plast Reconstr Surg. $2001 ; 107(4): 948-54$.

8. Saad MN, Kay S. The circumareolar incision: a useful incision for gynaecomastia. Ann R Coll Surg Engl. 1984;66(2):121-2.

9. Plourde PV, Reiter EO, Jou HC, Desrochers PE, Rubin SD, Bercu BB, et al. Safety and efficacy of anastrozole for the treatment of pubertal gynecomastia: a randomized, double- blind, placebo-controlled trial. J Clin Endocrinol Metab. 2004;89(9):4428-33.

10. Couke PA, Jichliski P, Matzinger O, Ozsahin M, Bulling SM, Zouhair A. Antiandrogen gynecomastia: an inescapable harm? Radiotherapy: a simple and efficient solution! Rev Med Suisse Romande. 2004;124(1):51-4.

11. Daniels IR, Layer GT. How should gynaecomastia be managed? ANZ J Surg. 2003;73(4):213-6.

12. Boljanovic S, Axelsson CK, Elberg JJ. Surgical treatment of gynecomastia: liposuction combined with subcutaneous mastectomy. Scand J Surg. 2003;92(2): 160-2.

13. Gruntmanis U, Braunstein GD. Treatment of gynecomastia. Curr Opin Investig Drugs. $2001 ; 2(5): 643-9$.

14. Tashkandi M, Al-Qattan MM, Hassanain JM, Hawary MB, Sultan $M$. The surgical management of high-grade gynecomastia. Ann Plast Surg. 2004;53(1):17-20.

15. Aslan G, Tuncali D, Terzioglu A, Bingul F. Periareolar-transareolarperithelial incision for the surgical treatment of gynecomastia. Ann Plast Surg. 2005;54(2):130-4.

16. Celebioglu S, Ertas NM, Ozdil K, Oktem F. Gynecomastia treatment with subareolar glandular pedicle. Aesthetic Plast Surg. 2004;28(5):281-6.

17. Bembo SA, Carlson HE. Gynecomastia: its features, and when and how to treat it. Cleve Clin J Med. 2004;71(6):511-7. 